\title{
Design of multilevel amplitude regenerative system
}

\author{
Mariia Sorokina \\ Aston Institute of Photonic Technologies, Aston University, B4 7ET Birmingham, UK (sorokinm@aston.ac.uk)
}

Received February 12, 2014; accepted March 8, 2014;

posted March 19, 2014 (Doc. ID 206384); published April 15, 2014

We propose a scheme for multilevel (nine or more) amplitude regeneration based on a nonlinear optical loop mirror (NOLM) and demonstrate through numerical modeling its efficiency and cascadability on circular 16-, 64-, and 256symbol constellations. We show that the amplitude noise is efficiently suppressed. The design is flexible and enables variation of the number of levels and their positioning. The scheme is compatible with phase regenerators. Also, compared to the traditional single-NOLM configuration scheme, new features, such as reduced and sign-varied power-dependent phase shift, are available. The model is simple to implement, as it requires only two couplers in addition to the traditional NOLM, and offers a vast range of optimization parameters. (c) 2014 Optical Society of America

OCIS codes: (060.2330) Fiber optics communications; (230.1150) All-optical devices; (230.4320) Nonlinear optical devices.

http://dx.doi.org/10.1364/OL.39.002499

The recent surge in multilevel all-optical regeneration is driven by dramatically increasing capacity demand, which requires the employment of advanced constellations [1]. All-optical regeneration offers an energyefficient solution for the removal of both deterministic and stochastic impairments, enabling high-capacity transmission [2-8]. A nonlinear optical loop mirror (NOLM) proposed in [9] was experimentally demonstrated as an efficient amplitude regenerator [ㄴ, $\underline{10}-\underline{12}]$, and its cascadability in long-haul transmission systems has been experimentally proved $[2, \underline{5}, \underline{13}, 14]$. However, the existing schemes are limited for up to two amplitude levels.

Here we propose and examine a novel scheme, which creates multiple regenerative (nine or more) levels in the amplitude transfer function (TF). This is achieved by coupling the NOLM transformed signal with the original wave. The scheme is flexible: additional parameters give more freedom for optimization-the number of levels and their positioning can be easily varied. We demonstrate the model on various circular 16- to 256-symbol constellations and observe efficient amplitude regeneration. Moreover, the scheme is compatible with phase regenerators, which can be relevant for applications in coherent optical communications.

The proposed regenerative scheme is depicted in Fig. 1. The initial signal $A_{\text {in }}=\sqrt{P_{\text {in }}} e^{i \varphi}$ is split by a $3 \mathrm{~dB}$ coupler with a transfer matrix given by

$$
\hat{\mathbf{C}}_{1}=\frac{1}{\sqrt{2}}\left(\begin{array}{ll}
1 & \mathrm{i} \\
\mathrm{i} & 1
\end{array}\right)
$$

Then one of the waves propagates through the NOLM element, which performs the following transformation:

$$
\hat{\mathbf{N}}\left(\sqrt{\frac{P_{\text {in }}}{2}}\right)=\left[-(1-\alpha) e^{i(1-\alpha) \gamma L P_{\text {in }} / 2}+\alpha e^{i \alpha \gamma L P_{\text {in }} / 2}\right] \sqrt{\frac{P_{\text {in }}}{2}}
$$

where $\alpha$ denotes the coupling ratio, $L$ is the length of the highly nonlinear fiber, and $\gamma$ is the fiber nonlinear coefficient. The other wave is phase rotated by $\Delta \phi=3 \pi / 2$ and undergoes the same propagation delay as the first one. Subsequently, the two waves are coupled by the second coupler, with the power ratio between them being $\kappa^{2}$. The latter is a significant parameter; it can be adjusted to the characteristic of the second coupler $\kappa=$ $\sqrt{\beta /(1-\beta)}$ (see the scheme in Fig. 1 ), where the transfer matrix is given by

$$
\hat{\mathbf{C}}_{2}=\left(\begin{array}{cc}
\sqrt{1-\beta} & \mathrm{i} \sqrt{\beta} \\
\mathrm{i} \sqrt{\beta} & \sqrt{1-\beta}
\end{array}\right),
$$

alternatively, having the second coupler be $1: 1$ and placing an attenuator after the NOLM and before the coupler. Of course, the two waves should propagate equal lengths to achieve balanced coupling. The obtained wave is amplified by $2 /(1-\beta)$ to restore the initial power. The resulting total TF is expressed via the initial field as

$$
\begin{aligned}
A_{\mathrm{out}}= & \sqrt{P_{\mathrm{out}}} e^{i(\varphi+\delta \phi)} \\
= & \left(\left[1+i \kappa-(1-\alpha) e^{i(1-\alpha) \gamma L P_{\mathrm{in}} / 2}\right.\right. \\
& \left.\left.+\alpha e^{i \alpha \gamma L P_{\mathrm{in}} / 2}\right]\right) \sqrt{P_{\mathrm{in}}} e^{i \varphi} .
\end{aligned}
$$

As a result of the transformation, the TF of the output power versus the initial power has multiple plateau regions around the given stationary and stable points of the transformation. In Fig. 2(a) we plotted the input power of the device normalized by $\gamma L / 2$; this defines a dimensionless nonlinear phase shift in the NOLM:

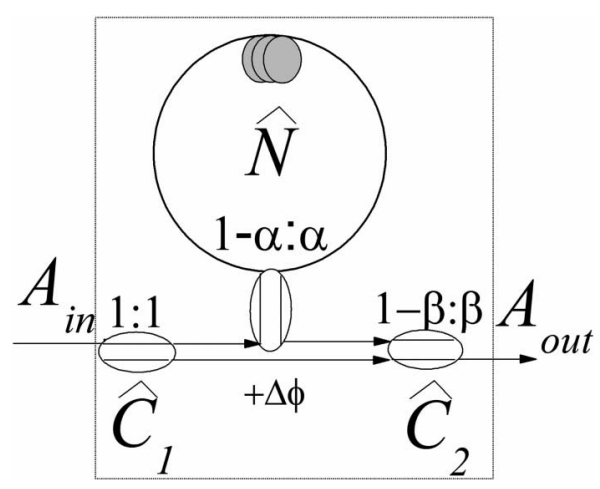

Fig. 1. Scheme of the coupled NOLM. 

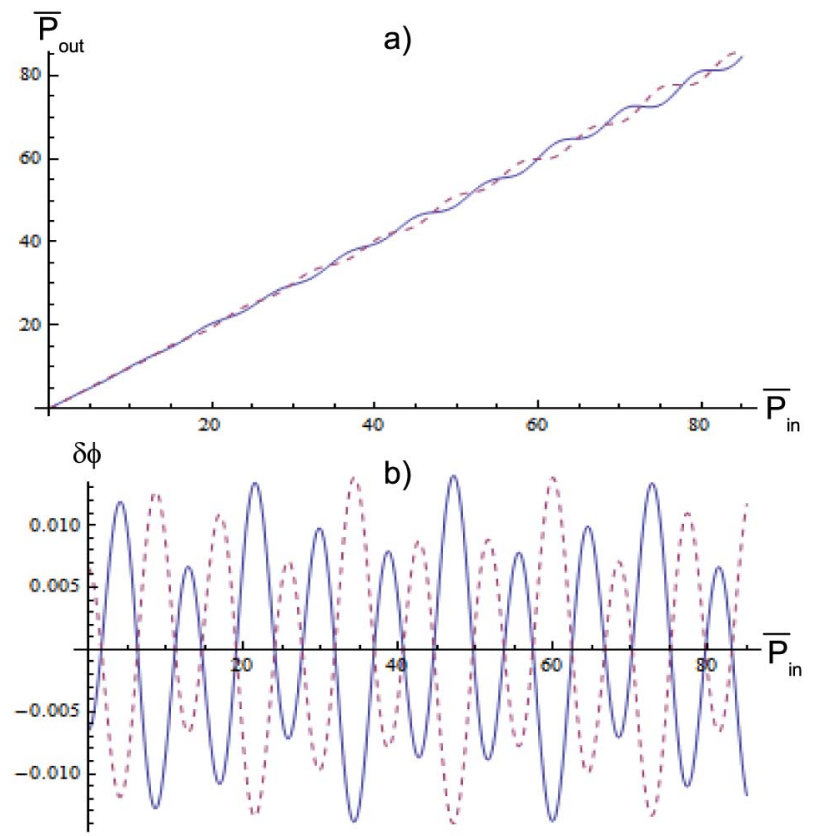

Fig. 2. (a) $\mathrm{TF}$ for the dimensionless power $\bar{P}=P \gamma L / 2$ and (b) associated phase shift for parameters $\alpha=0.267$; $\Delta \phi=3 \pi / 2 ; \kappa=\sqrt{\beta /(1-\beta)}=0.014$ (solid curves). By changing parameters to $\alpha^{\prime}=1-\alpha$, one can achieve sign-reversed phase shift, and the amplitude levels will be shifted (dashed curves).

$\bar{P}_{\text {in }}=P_{\text {in }} \gamma L / 2$. By changing the fiber length and nonlinearity coefficient, one can adjust the value of the input power as desired. In particular, for $L=1 \mathrm{~km}$ and $\gamma=10(\mathrm{~W} \cdot \mathrm{km})^{-1}[\underline{10}]$, the average input power (for equiprobable symbols) is $32.4,35.7$, and $39.3 \mathrm{dBm}$ for circular 16-, 64-, and 256-quadrature amplitude modulation (QAM) constellations, respectively (shown in Fig. 3). Note that nonlinear fibers with higher values of $\gamma$ (e.g., bismuth fibers) are currently commercially available, which will further relax the demand on the signal power requirement. Moreover, the number and positioning of the levels can be easily varied by adjusting TF parameters $\alpha$ and $\kappa$ by applying the optimization procedure of [15] to the amplitude and phase response:

$$
\begin{aligned}
P_{\text {out }}= & P_{\text {in }}\left(1+\kappa^{2}\left((1-\alpha)^{2}+\alpha^{2}\right)-2 \kappa^{2}(1-\alpha) \alpha \cos [(1-2 \alpha) \psi]\right. \\
& +2 \kappa[(1-\alpha) \sin [(1-\alpha) \psi]-\alpha \sin [\alpha \psi]]), \\
\psi= & \frac{\gamma L P_{\text {in }}}{2}, \\
\delta \phi= & \arctan \left(\frac{\alpha \kappa \cos (\alpha \psi)-(1-\alpha) \kappa \cos ((1-\alpha) \psi)}{1-\alpha \kappa \sin (\alpha \psi)+(1-\alpha) \kappa \sin ((1-\alpha) \psi)}\right) .(2)
\end{aligned}
$$

Also, the transformation is accompanied by an additional power-dependent phase shift [see Fig. 2(b)], which is reduced compared with the traditional oneNOLM scheme. Moreover, since the phase shift is sign varied, by changing the parameters one can easily cause the phase shift to be sign reversed. In particular, if one chooses $\alpha^{\prime} \rightarrow 1-\alpha$ or, alternatively, $\kappa^{\prime} \rightarrow-\kappa$ (or $\left.\Delta \phi^{\prime} \rightarrow \Delta \phi+\pi\right)$, then a new phase shift will be sign reversed [see Fig. 2(b)], and the stable points of the amplitude TF will be shifted [see Fig. 2(a)] to a different set of the same stationary points: $T\left(P_{\text {in }}\right)=P_{\text {in }}$. This highlights the importance of the optimization requirements of [15] (stability and stationarity) to be completely fulfilled for efficient regeneration. The dimensionless output power $\bar{P}_{\text {out }}$ and phase shift $\delta \phi$ versus the normalized initial power $\bar{P}_{\text {in }}$ for parameters $\alpha=0.267$ and $\kappa=0.014$ are shown in Figs. 2(a) and 2(b), respectively.

Next we demonstrate through numerical modeling the regenerative effect of the proposed scheme by simulating numerically the transmission of circular 16-, 64-, and 256QAM via a noisy channel. The amplitude regenerators are placed equidistantly in cascades along the line. Noise is modeled as additive white Gaussian noise uniformly distributed along the line. The transmission between the spans is assumed to be linear. Here we focus on the demonstration of the regenerative properties and neglect nonlinear and dispersive effects in-line. For benchmarking we use the corresponding linear system (the respective channel in the absence of regeneration) and characterize it by the signal-to-noise ratio (SNR) of the linear channel. The performance was characterized by the symbol error rate (SER) obtained by direct error counting of $2^{25}$ simulated equiprobable symbols.

In the circular symbol constellations the amplitude levels were defined by the stationary and stable points of the amplitude $\mathrm{TF}$ (determined by the method of [15]), whereas the phase distribution was chosen from the analogues 16-, 64- and 256- circular QAM [16-18]. Though the constellations are nonoptimal for the linear channel, and in the absence of regeneration their performance is worse than the optimized circular or rectangular QAM, the effective amplitude noise suppression enables us to improve performance and significantly outperform the SER of the corresponding linear channel. Further simultaneous optimization of the input format and $\mathrm{TF}$ parameters will increase performance. The results demonstrate the importance of optimization of signal packing for regenerative channels [19].

The power-dependent phase shift, $\delta \phi$, though it is small, can be significant for densely packed constellations in the cascaded regime. It can be reduced by an optical phase conjugator (OPC) inserted in the middle of the transmission line (this was assumed in the simulations) or by the consequent regenerator (as the sign of the phase shift can be inverted by a different set of parameters). Alternatively, one can employ phase regenerators, which will remove the phase shift as well as causing a reduction in the phase noise (as demonstrated in the next section).

Figure $\underline{3}$ shows constellations at the receiver and the improvement in the SER after transmission in a linear channel without regenerators (blue, left) and in a regenerative channel with 10 (green, center) and 20 (red, right) regenerators. By varying the parameters, we change the number of the regenerative levels and their positioning. The scheme is flexible and able to handle different formats. Increasing the number of regenerators in the cascade above the critical number leads to the saturation effect. Thus, we have illustrated that the amplitude noise is effectively squeezed, whereas the residual phase noise affects the SER. To improve system performance further, phase regeneration is required. 

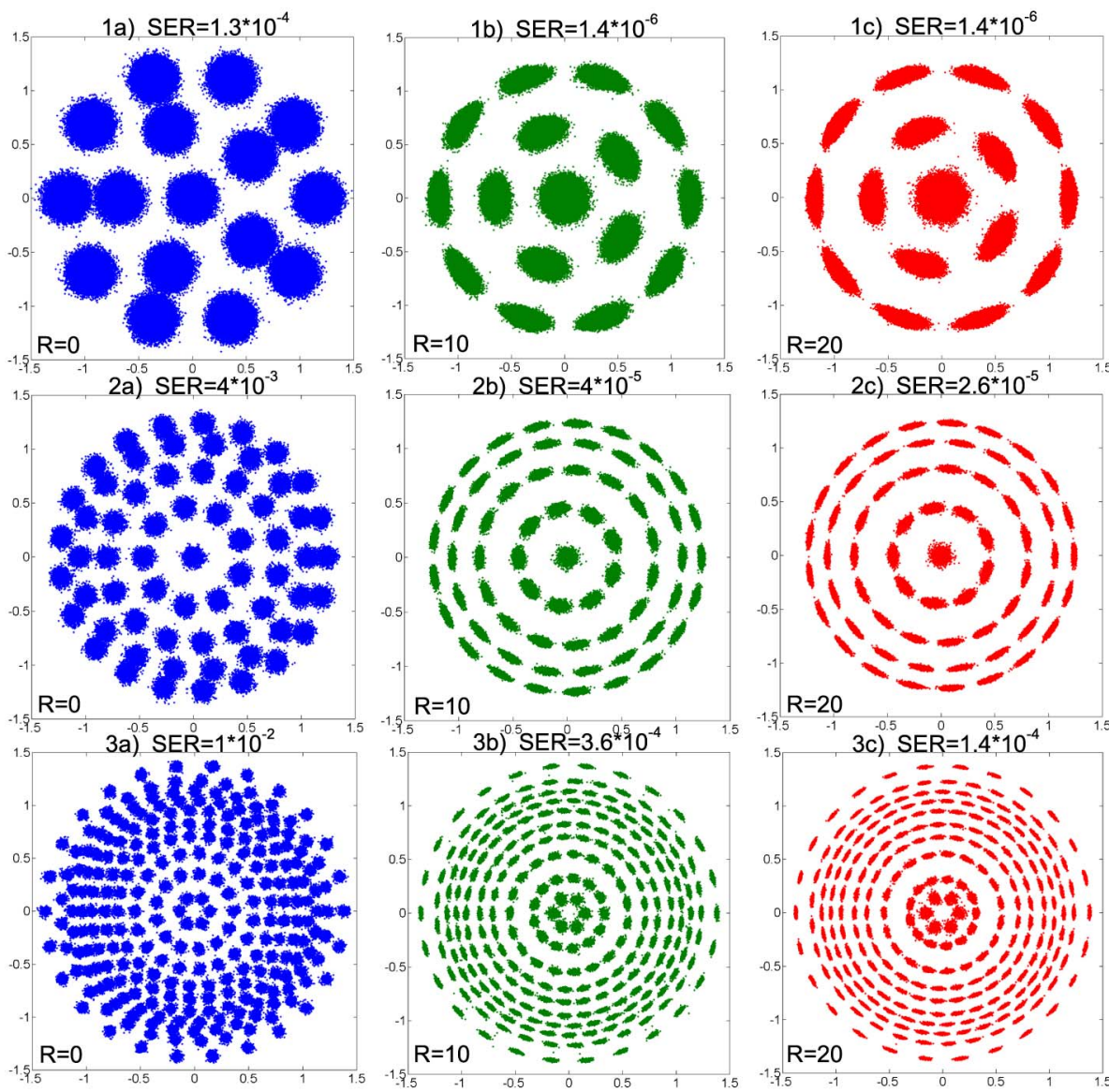

Fig. 3. Constellation diagrams (normalized to signal power $S=1$ ) for circular 16- (row 1), 64- (row 2), and 256- (row 3) QAM after transmission without (column a) and with 10 (column b) and 20 (column c) equidistantly placed amplitude regenerators [see Fig. 1 with $\gamma L=2 \mathrm{~W}^{-1} ; \alpha=0.24, \kappa=0.03$ (rows 1 and 2) and $\alpha=0.267, \kappa=0.014$ (row 3)], with an OPC placed in the middle of the transmission line. In the absence of regeneration, the respective linear system has $\mathrm{SNR}=20 \mathrm{~dB}($ row 1 ), $\mathrm{SNR}=25 \mathrm{~dB}(\mathrm{row}$ 2 ), and SNR $=30 \mathrm{~dB}$ (row 3).
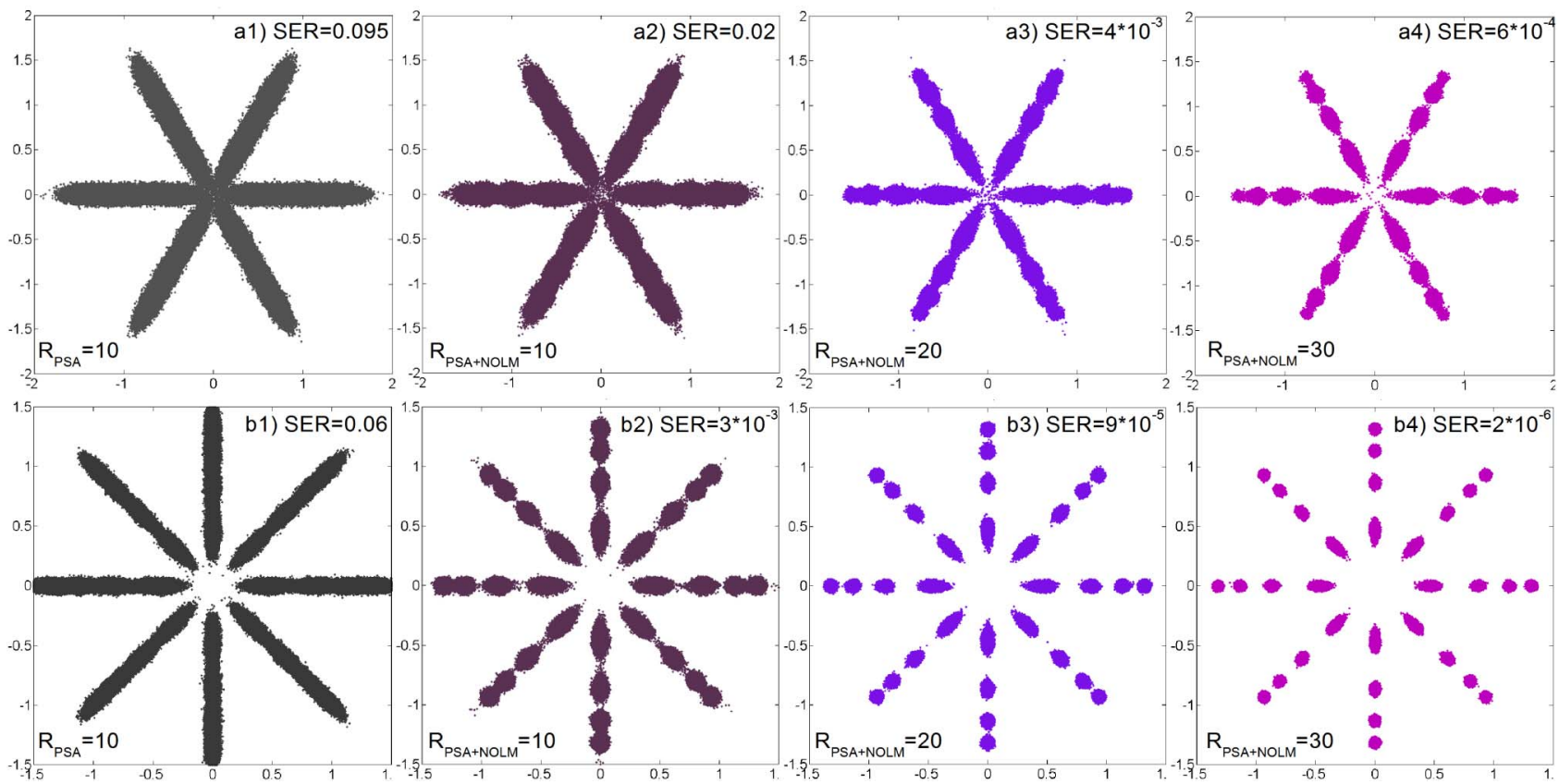

Fig. 4. Constellation diagrams (normalized to signal power $S=1$ ) for circular 16- (row a) and 32- (row b) QAM after transmission with 10 PSAs (column 1) and 10,20, and 30 (columns 2-4, respectively) phase and amplitude regenerators equidistantly placed along the line (with parameters $\gamma L=2 \mathrm{~W}^{-1}, \alpha=0.24, \kappa=0.03$ ). In the absence of regeneration, the respective linear system has SNR $=$ $15 \mathrm{~dB}$ (row a) and $\mathrm{SNR}=20 \mathrm{~dB}$ (row b). 
Next we demonstrate that the proposed scheme is compatible with phase regenerators. In particular, we further consider a phase quantizer based on a phasesensitive amplifier (PSA) placed after each amplitude regenerator. The $\mathrm{TF}$ of the M-level phase quantizer [20] (the cascadability of which was demonstrated in []] is given by

$$
\sqrt{P_{\text {out }}} e^{i \varphi_{\text {out }}}=\sqrt{P_{\text {in }}} e^{i \varphi}\left(1+m e^{-i M \varphi}\right) .
$$

The amplitude ratio between the signal and its $M-1$ harmonic was put to its optimal value: $m=(M-1)^{-1}$ [15]. Here we consider six- and eight-level PSAs $[20,21]$. Figure 4 shows the constellation diagrams received after 10, 20 , and 30 combined phase and amplitude regenerators (for reference we also plotted the constellation received after 10 PSAs). One can see that the simultaneous suppression of the amplitude and phase noise is achieved for multilevel formats.

We proposed a novel scheme for multilevel amplitude regeneration and demonstrated effective amplitude noise suppression for nine (or more) amplitude levels. The design is simple and flexible, in that by varying parameters one can change the number of levels and adjust their positioning. The model was illustrated on circular 16-, 64- and 256-QAM. Combined with PSAs, the simultaneous suppression of both phase and amplitude noise was achieved and demonstrated on circular 16- and 32-QAM.

We are grateful to S. K. Turitsyn, S. Sygletos, and A. D. Ellis for useful discussions. This work has been supported by the EPSRC project UNLOC (Unlocking the Capacity of Optical Communications) EP/J017582/1.

\section{References}

1. P. J. Winzer and R.-J. Essiambre, J. Lightwave Technol. 24, 4711 (2006).

2. M. Nakazawa, E. Yamada, H. Kubota, and K. Suzuki, Electron. Lett. 27, 1270 (1991).
3. O. Leclerc, U. E. Desurvire, and O. Audouin, Opt. Fiber Technol. 3, 97 (1997).

4. S. Boscolo, S. K. Turitsyn, and K. J. Blow, Opt. Fiber Technol. 14, 299 (2008).

5. M. Matsumoto, IEEE J. Sel. Top. Quantum Electron. 18, 738 (2012).

6. K. S. Turitsyn and S. K. Turitsyn, Opt. Lett. 37, 3600 (2012).

7. M. A. Sorokina and S. K. Turitsyn, "Shannon capacity of nonlinear regenerative channels," arXiv:1305.1537 (2013).

8. T. Umeki, M. Asobe, H. Takara, Y. Miyamoto, and H. Takenouchi, Opt. Express 21, 18170 (2013).

9. N. J. Doran and D. Wood, Opt. Lett. 13, 56 (1988).

10. A. G. Striegler, M. Meissner, K. Cvecek, K. Sponsel, G. Leuchs, and B. Schmauss, IEEE Photon. Technol. Lett. 17, 639 (2005).

11. T. I. Lakoba and M. Vasilyev, Opt. Express 15, 10061 (2007).

12. T. Roethlingshoefer, G. Onishchukov, B. Schmauss, and G. Leuchs, IEEE Photon. Technol. Lett. 26, 556 (2014).

13. F. Seguineau, B. Lavigne, D. Rouvillain, P. Brindel, L. Pierre, and O. Leclerc, Proceedings of the Optical Fiber Communication Conference (2004), paper WN4.

14. A. Gray, Z. Huang, I. Khrushchev, and I. Bennion, Electron. Lett. 40, 498 (2004).

15. M. A. Sorokina, S. Sygletos, and S. K. Turitsyn, Opt. Lett. 38, 4378 (2013).

16. G. J. Foschini, N. J. Holmdel, R. Gitlin, and S. Weinstein, IEEE Trans. Commun. 22, 28 (1974).

17. Z. H. Peric, I. B. Djordjevic, S. M. Bogosavljevic, and M. C. Stefanovic, 9th Mediterranean Electrotechnical Conference (1998), Vol. 2, pp. 866-869.

18. R. Dischler, Optical Fiber Communication Conferencel National Fiber Optic Engineers Conference, OSA Technical Digest (online) (Optical Society of America, 2013), paper OTh3B.2.

19. M. Sorokina, S. Sygletos, A. D. Ellis, and S. Turitsyn, Opt. Express 21, 31201 (2013).

20. J. Kakande, R. Slavik, F. Parmigiani, A. Bogris, D. Syvridis, L. Gruner-Nielsen, R. Phelan, P. Petropoulos, and D. J. Richardson, Nat. Photonics 5, 748 (2011).

21. G. Hesketh and P. Horak, Opt. Lett. 38, 5357 (2013). 